\title{
Impacto da amamentação e da obesidade parental na saúde infantil
}

\author{
Impact of breastfeeding and parental obesity in child health
}

\section{AUTORES \\ Anelise Bozzetto da Silva ${ }^{1}$ (D) \\ Karine Elisa Schwarzer Schmidt ${ }^{1}$ (D) \\ Luciane Durigon Cocco $^{1}$ (D) \\ 1 Programa de Pós-Graduação em Ciências da Saúde: Cardiologia. Instituto de Cardiologia do Rio Grande do Sul / Fundação Universitária de Cardiologia. Porto Alegre, Rio Grande do Sul, Brasil. \\ CONTATO \\ Karine Elisa Schwarzer Schmidt karine_schmidt@hotmail.com \\ Programa de Pós Graduação em Ciências da Saúde: Cardiologia. Instituto de Cardiologia do Rio Grande do Sul/Fundação Universi- tária de Cardiologia. \\ Avenida Princesa Isabel, 370, Santana, Porto Alegre, Rio Grande do Sul, Brasil. CEP: 90620-000. \\ DOI}

10.12820/rbafs.23e0058

\section{(cc) BY-NC-SA}

Este obra está licenciado com uma Licença Creative Commons Atribuição-NãoComercialCompartilhalgual 4.0 Internacional.

\section{Prezado Editor,}

O artigo "Associação do estado nutricional e aptidão cardiorrespiratória com a prática de atividade física e indicadores de obesidade familiar em escolares"1 despertou-nos especial interesse e reflexão. A sua ampla abordagem ao unir aptidão cardiorrespiratória (APCR) e índice de massa corporal (IMC), considerando indicadores de obesidade familiar, peso ao nascer e histórico de amamentação, merece destaque.

Dentre os resultados apresentados, chama-nos atenção que o baixo peso ao nascer não tenha sido associado com baixa APCR/excesso de peso, sendo essa relação encontrada apenas em crianças nascidas com peso normal e elevado, o que, conforme indicado pelos autores, contradiz resultados da literatura, que mostram que as nascidas com baixo peso ou excesso de peso tendem a exibir baixa APCR e aumento de peso ${ }^{1}$. Surgiu, a partir disso, o interesse em saber o nível sócioeconômico dessa população, pois, conforme comentado na discussão do manuscrito, essa variável pode atuar como um fator de confusão, considerando que o baixo peso ao nascer é mais prevalente em populações empobrecidas e em desenvolvimento, enquanto o elevado peso ao nascer apresenta prevalência aumentada em alguns países desenvolvidos ${ }^{2}$. Os autores descrevem nos métodos que a classe econômica foi mensurada através do critério da Associação Brasileira de Empresas de Pesquisa (ABEP), contudo, a classe econômica não foi descrita claramente nos resultados de caracterização da amostra, apenas foi referida nos ajustes, e talvez fosse uma informação importante a ser acrescida para enriquecer ainda mais os achados. Reforçamos a importante consideração feita por Moraes et al. ${ }^{1}$, que, devido aos valores do peso ao nascimento terem sido referidos pelos próprios pais ou responsáveis através de questionário, pode haver um viés de informação e de memória, o que salientamos que pode ser acentuado pelo baixo esclarecimento, associado a classes econômicas mais baixas.

Moraes et al. ${ }^{1}$ reportaram que, até aquele momento, não haviam sido encontrados estudos que investigassem a associação entre amamentação e APCR. Encontramos, contudo, um estudo na literatura com 1025 crianças e 971 adolescentes da Estônia e Suécia ${ }^{3}$, que complementa os achados de Moraes et al. ${ }^{1}$, quanto aos escolares que receberam amamentação exclusiva por leite materno até os seis meses apresentarem melhores níveis de APCR e estado nutricional. Este estudo também demonstrou que maior duração da amamentação foi associada com maior APCR, independentemente de 
fatores de confusão (país, sexo, idade, estado puberal e IMC). Entretanto, diferentemente do estudo de Moraes et al. ${ }^{1}$ o IMC do pai e dos avós não foram avaliados ${ }^{3}$.

Moraes et al. ${ }^{1}$ comentam em sua discussão sobre a escassez de estudos associando a APCR de crianças e adolescentes com o histórico de obesidade familiar. $\mathrm{O}$ estudo longitudinal de base populacional Northern Finland Birth Cohort $1986^{4}$ almejou verificar como os determinantes pré-natais estão associados com atividade física e APCR na adolescência. Foi demonstrado que maior peso ao nascer e maior tempo de gestação predisseram menores níveis de atividade física e de APCR aos 16 anos. Sobrepeso/obesidade da mãe ou do pai antes da gravidez foram associados a níveis mais baixos de atividade física e aptidão física da criança na adolescência. Contudo, após ajustes para os transtornos da gravidez materna e para o IMC do adolescente, as associações com o sobrepeso/obesidade da mãe foram atenuadas, mas não as do $\mathrm{pai}^{4}$, semelhantemente ao estudo de Moraes, no qual a obesidade da mãe, da avó ou avô materno não se associaram a variável APCR/IMC, apenas a obesidade paterna ${ }^{1}$.

Os autores levantaram a hipótese de que escolares que apresentassem um histórico familiar de obesidade teriam tendência a apresentar um estilo de vida mais inativo, uma vez que adultos obesos tendem a praticar menos atividades físicas e passar mais tempo em atividades sedentárias ${ }^{1}$. Essa hipótese é reforçada por um estudo transversal que examinou a associação entre IMC dos pais e composição corporal com aptidão física e fatores de estilo de vida dos filhos (tempo sedentário, atividade física e dieta), e que constatou que maiores valores de IMC materno e paterno foram relacionados a menores escores no teste do salto em comprimen- to e maiores níveis de sedentarismo, respectivamente ${ }^{5}$. Contudo, estudos longitudinais avaliando as relações entre essas variáveis ainda são necessários.

Dessa maneira, embora seja um estudo transversal, Moraes et al. $^{1}$ trouxeram importantes contribuições sobre a complexidade da associação do IMC e APCR com a prática de atividade física e indicadores de obesidade familiar em escolares. A interlocução de diversas variáveis na saúde infantil é relevante e serve de modelo para futuras pesquisas sobre APCR e IMC em escolares, especialmente estudos de coorte, ainda escassos na literatura sobre essa temática.

\section{Referência}

1. Moraes G, Tornquist D, Tornquist L, Reuter C, Burgos M. Associação do estado nutricional e aptidão cardiorrespiratória com a prática de atividade física e indicadores de obesidade familiar em escolares. Rev Bras Ativ Fís Saúde. 2017;22(6):540-45.

2. Rossi C E, Vasconcelos F A G. Peso ao nascer e obesidade em crianças e adolescentes: uma revisão sistemática. Rev Bras Epidemiol. 2010;13(2):246-58.

3. Labayen I, Ruiz JR, Ortega FB, Loit HM, Harro J, Villa I, et al. Exclusive breastfeeding duration and cardiorespiratory fitness in children and adolescents. Am J Clin Nutr. 2012;95(2):498-505.

4. Tikanmäki M,Tammelin T, Vääräsmäki M, Sipola-Leppänen M, Miettola S, Pouta A, et al. Prenatal determinants of physical activity and cardiorespiratory fitness in adolescenceNorthern Finland Birth Cohort 1986 study. BMC Public Health. 2017;17(1):346.

5. Cadenas-Sanchez C, Henriksson P, Henriksson H, Nyström $\mathrm{CD}$, Pomeroy J, Ruiz J, et al. Parental body mass index and its association with body composition, physical fitness and lifestyle factors in their 4-year-old children: results from the MINISTOP trial. Eur J Clin Nutr. 2017;71(10):1200.

Recebido: $21 / 08 / 2018$ Aprovado: 13/05/2019 